\title{
COMPARISON OF METFORMIN AND PIOGLITAZONE IN PCOS
}

Archana V. Rokade, Javdekar D. P, Patange R. P.

1. Assistant Professor. Department of Obstetrics \& Gynaecology, Krishna Institute of Medical Sciences \& deemed University, Karad.

2. Assistant Professor. Department of Obstetrics \& Gynaecology, Krishna Institute of Medical Sciences \& deemed University, Karad.

3. Professor \& HOD. Department of Obstetrics \& Gynaecology, Krishna Institute of Medical Sciences \& deemed University, Karad.

\section{CORRESPONDING AUTHOR:}

Dr. Archana V. Rokade,

70, seta Shankar,

Swami Samartha Prasad,

Shaniwar Peth, Karad,

Dist- Satara-415110.

E-mail: dr.archanarokade@gmail.com

ABSTRACT: OBJECTIVES: To compare and evaluate the effects of metformin and pioglitazone on insulin resistance, ovulation and hyperandrogenism in women with PCOS. METHODS: Total 180 patients were included in this double blind controlled trial between 18 to 30 years. These women were randomly allocated in two groups. After taking written consent they were treated with either metformin or pioglitazone for 6 months and pretreatment versus post treatment clinical and biochemical variables were analysed and compared. RESULTS: After treatment with metformin and pioglitazone around 50- $55 \%$ women in both group achieved menstrual cycle regularity, and ovulation was restored in $44 \%$ and $55 \%$ in patients on metformin and pioglitazone respectively. Both group showed decline in F-G score. Decrease in serum cholesterol was seen in both groups, but was more pronounced with pioglitazone. Effect on insulin resistance and hyperinsulinemia was assessed by measuring pre and post treatment fasting serum insulin levels . Fall in serum insulin level was more with pioglitazone and $48 \%$ women became normoinsulinemic after treatment with pioglitazone as compared to $12 \%$ with metformin. Rise in serum SHBG and fall in LH level was more remarkable with pioglitazone ( $p$ value <0.05).

CONCLUSION: Pioglitazone has remarkable beneficial effect on hyperinsulinemia, hyperandrogenism and ovulation in women with PCOS.. Along with this metabolic and reproductive benefit it has preventive action on risk of development of type 2 diabetes and cardiovascular problems. So it may come up as new treatment modality for metabolic correction in PCOS.

INTRODUCTION: Polycystic ovarian syndrome being heterogenous disorder affect $7-10 \%$ of population ${ }^{1,2}$. It is spectrum of disorders with mild presentation in some and severe reproductive and metabolic disturbances in others, PCOS is characterized by chronic anovulation or hypo ovulation, hyperandrogenism ( clinical or biochemical), and or on ultrasonography cystic enlarged ovaries. Peri or post pubertal obesity, insulin resistance and consequent hyperinsulinemia are important co- morbidities of PCOS and can aggrevate hyperandrogenism. Data that support pathogenic role of hyperinsulinemia as

1. At central level insulin causes deregulation of LH secretion, 
2. At ovarian level it enhance P450c17 enzyme and lead to intraovarian hyperandrogenism that affect follicular growth

3. Insulin decreases sex hormone binding globulin synthesis and increases free androgen levels

4. Promote ACTH stimulated adrenal androgen secretion.

Women with PCOS and insulin resistance have high risk ${ }^{3}$ of cardiovascular accidents due to hypertension, dyslipidemia and increased risk of atherosclerosis and so ischemic heart disease. They also have increased risk of type 2 diabetes and endometrial and breast cancer due to increased testosterone and unopposed estrogen.

Thus relation between insulin and androgen excess has stimulated efforts to either decrease in secretion of insulin or improvement in action of insulin at target tissue which can help in removing stigmata of hyperandrogenism leading to metabolic correction and improvement in reproductive function. Along with lifestyle changes which include dietary and exercise regimen , insulin sensitizers are emerging as new and rational treatment modality.

STUDY DESIGN: Randomized double blind comparative study conducted in Krishna institute of medical sciences and deemed university over a period of 6 months. Women between age of 1830 yrs were included and randomly allocated in two groups. After complete evaluation by history, physical examination and investigation group 1 was given tab metformin $500 \mathrm{mg}$ BD and GROUP 2 was treated with tab pioglitazone $7.5 \mathrm{mg}$ BD. Each group consisted of 80 patients.

\section{MATERIALS AND METHODS:}

\section{Exclusion criteria-}

1 Pregnancy and lactation

2 Impaired liver and renal function tests

3 Thyroid disorder

4 Hyperprolactinemia

5 Adrenal disorders

6 Patient on estrogen and progesterone pills

Inclusion criteria- age - 18 to 30 yrs diagnosed PCOS by ESHRE/ ASRM consensus criteria4-

1. Oligo or anovulation

2. Clinical or biochemical hyperandrogenism

3. Sonography suggestive of polycystic ovaries ( ovarian volume $>10 \mathrm{cc}, 12$ or more follicles of 2-9 $\mathrm{mm}$ )

After taking written consent study conducted in follicular phase of spontaneous or induced (Medroxyprogesterone acetate) menstrual cycle. History of menstrual cycle taken , BMI calculated physical examination done to rule out galactorrhea and thyroid swelling. Hirsutism graded by FERRIMAN GALLWEY score. After this evaluation blood sample taken for 1 CBC, 2 liver and renal function test,3. Thyroid function test, $4 \mathrm{sr}$. prolactin, $5 \mathrm{sr}$ fasting insulin level, 6 LH/ FSH ratio, 7. ( SHBG) sex hormone binding globulin, 8.sr. cholesterol.

Patients were followed every monthly. After 6 months of treatment efficacy of metformin and pioglitazone compared by evaluating following parameters 1 . Menstrual regularity 2. Decrease in F-G Scores 3 fasting insulin level 4. decline in LH levels 5. Decline in LH/ FSH ratio 6.ovulation. 7.SHBG, 8.Sr. cholesterol levels 
Standard statistical $\mathrm{z}$ test based probability was derived by comparing means and $\mathrm{P}<$ 0.05 was taken statistically significant. This was calculated for all variables and baseline and post treatment values compared in both groups. Confidence level for each mean was $95 \%$.

\section{Observations and results-}

\section{Clinical characteristics-}

Menstrual regularity- In group1 seventy eight were having menstrual irregularity initially and at end of 6 months 36 reported regular menstrual cycle .(46\%) in group 2 seventy nine were having irregular menstrual cycle at beginning of study and at the end of 6 months 45 reported to have regular menstrual cycle ( $58 \%$ ).

Virilisation- In group 1 fifty were having hirsutism with F-G score more than 8 and all of them reported lesser F-G score at end of6 months. In group 2 out of 80, fifty two had F-G score more than 8 at beginning of study and all reported lesser FG score at end of study.

\section{Biochemical parameters:}

Serum fasting insulin-In group 1 out of eighty, 44 (55\%) and in group 2 fifty seven $(70 \%$ ) were having hyperinsulinemia ( fasting insulin level $>40 \%$ ) at start of study. At end of study fall in insulin level was more in group 2 ( from $42 \mathrm{uU} / \mathrm{ml}$ to $21 \mathrm{uU} / \mathrm{ml}$ ) as compared to group 1 ( from 40 to $34 \mathrm{uU} / \mathrm{ml}$ ).

Number of patients becoming normoinsulinemic after treatment were also more in group 2( $48 \%$ ) as compared to group 1 (12\%).

LH value- In group $1 \mathrm{LH}$ value on an average decreased by $2.31 \mathrm{~m} \mathrm{IU} / \mathrm{ml}$ and in group 2 decreased by $4.6 \mathrm{~m} \mathrm{IU} / \mathrm{ml}$ which is statistically significant versus baseline values.

LH/ FSH ratio- Fall in LH/ FSH ratio was significant and more patient showed this decline in group 2 as compared to group 1

Serum cholesterol level- In group 1 out of 80, eleven had serum cholesterol > $200 \mathrm{mg} / \mathrm{dl}$ at baseline and at end of study only eight remained hyperlipidemic while in group 2 out of fourteen only four remained with hypercholesterolemia after 6 months of treatment with pioglitazone.

SHBG-In both groups there was rise in sex hormone binding globulin as compared to baseline and rise was statistically significant with pioglitazone.

Ovulation- In group 1 out of 78 , thirty four restored ovulation (45\%) whereas in group 2 out of 79 , forty three(55\%) restored ovulation at end of 6 months of study.

Side effect profile- Group 1 mild gastrointestinal symptoms reported but didn't required discontinuation of treatment. In group 2 mild peripheral edema in $40 \%$ and muscle cramping noted in $11 \%$,but no deranged hepatic function observed.

TABLE1- Comparison of baseline values in group 1 and group2-

\begin{tabular}{|l|l|l|l|}
\hline Variables & Group 1 & Group 2 & P values \\
\hline F-G grading & $15.6+/-5.80$ & $14.32+/-5.27$ & $<0.05$ \\
\hline Sr cholesterol $(\mathrm{mg} / \mathrm{dl})$ & $182.6+/-14.3$ & $188.5+/-10$ & $<0.05$ \\
\hline Fasting insulin level $(\mathrm{uU} / \mathrm{ml})$ & $43+/-7.4$ & $42+/-8.9$ & $<0.05$ \\
\hline SHBG $(\mathrm{nmol} / \mathrm{L})$ & $45+/-16$ & $32.1+/-10.5$ & $<0.05$ \\
\hline LH $(\mathrm{mIU} / \mathrm{ml})$ & $4.5+/-1.1$ & $6.1+/-4.1$ & $<0.05$ \\
\hline LH/ FSH & $0.68+/-0.1$ & $0.94+/-0.27$ & $<0.05$ \\
\hline
\end{tabular}


TABLE 2- Comparison of post treatment values of group 1 and group2

\begin{tabular}{|l|l|l|l|l|}
\hline VARIABLES & Group 1 & Group2 & Z scores & P values \\
\hline F-G grading & $7.81+/-4.2$ & $7.88+/-5.5$ & 0.65 & $>0.05$ \\
\hline Total cholesterol(lmg/dl) & $172+/-12.13$ & $149.5+/-10.7$ & 9.543 & $<0.05$ \\
\hline $\begin{array}{l}\text { Fasting insulin } \\
(\mathrm{u} \mathrm{U} / / \mathrm{ml})\end{array}$ & $36.9+/-5.7$ & $21.3+/-3.2$ & 14.65 & $<0.05$ \\
\hline SHBG & $36.67+/-9.1$ & $80.44+/-16.21$ & 15.42 & $<0.05$ \\
\hline LH & $4.7+/-2.5$ & $2.1+/-0.9$ & 6.2 & $<0.05$ \\
\hline
\end{tabular}

DISCUSSION: With both metformin and pioglitazone we observed that nearly $50 \%$ of patients achieved ovulation and regular cycle. A decline in F-G grading for hirsutism was seen in both groups and decrease in serum cholesterol was also seen in both groups but significant with pioglitazone in group 2. Fall in testosterone levels and rise in SHBG were observed.

Fall in LH is seen in both groups but significant with pioglitazone. Some studies have demonstrated fall in LH and LH/FSH ratio only in obese persons. ${ }^{5}$

In our study $63.4 \%$ people were hyperinsulinemic supporting hypothesis ${ }^{6}$ that that hyperinsulinemia plays important role in pathogenesis of PCOS. . The two insulin sensitizers had beneficial effects on lipid profile, and amelioration of insulin resistance indices. The results of our study indicates that amongst these two different insulin sensitizers, metformin and pioglitazone, greater improvement in insulin resistance indices (ISI and glucose-to-insulin ratio) with pioglitazone. Buchannan et al showed that administration of pioglitazone reduced the incidence of diabetes mellitus by more than $50 \%$ and protection persisted even after medication was stopped.

In study by Romualdi et al decrease in LH level and LH/FSH ratio was clearly demonstrated in both normoinsulinemic and hyperinsulinemic PCOS patients, and decrease was statistically significant after treatment with pioglitazone. Pioglitazone is insulin sensitizer by its action on nuclear receptors PPAR gamma ${ }^{7}$ which lead to increased production of insulin sensitive adipocytes and increased glucose uptake in peripheral tissue. Even it has additional beneficial action on hyperandrogenism, lipid profile and anovulation. All this effects are independent of BMI and insulin secretion ${ }^{8}$.

Seto-Young colleague's ${ }^{9}$ have described that PPAR- gamma agonist causes increased production of progesterone and decrease in testosterone and estrogen and concurrently antagonize insulin mediated rise in testosterone in cultured human ovarian tissue. Even Pioglitazone also ameliorate sign and symptoms of PCOS in women cohort who have failed to respond to metformin ${ }^{10}$,

One study provides clear evidence of early impairment of endothelial function in young women with PCOS who are not dyslipidemic or hypertensive compared with age- and BMImatched controls in accordance with previous data11,12,13.

Metformin administration for 6 months in women with PCOS induced a significant increase in flow-mediated dilation that was restored to normal values with a concomitant decrease in insulin resistance and hyperandrogenism indices ${ }^{14}$.Pioglitazone administration for 6 months in women with PCOS also resulted in an increase in flow-mediated dilation and an improvement in insulin resistance and hyperandrogenism indices.. In women with PCOS, pioglitazone administration has been shown to reduce soluble markers of atherosclerosis and indices of insulin resistance and hyperandrogenism ${ }^{15}$. 
In conclusion, young women with PCOS have impaired endothelial function indicating an increased risk for early onset cardiovascular disease ${ }^{16}$. Treatment with metformin or pioglitazone for 6 months induces a similar beneficial effect on endothelial function that is restored to normal values. This may be attributed partially to an improvement in insulin resistance, and other metabolic and nonmetabolic actions of insulin sensitizers also may be contributing. Long-term studies are needed to investigate whether treatment with insulin sensitizers also may improve cardiovascular prognosis in women with PCOS ${ }^{17}$.

Thus according to Geller et al pioglitazone may give reproductive, metabolic and cardiovascular benefit to women with PCOS who have failed to respond to metformin. And our study have confirmed the same findings.

At the same time thiazolidinediones have to be stopped as soon as patient is pregnant as they are category $\mathrm{C}$ drug by FDA and they have teratogenic risk, on the contrary metformin being category B drug it is safe in pregnancy and reduces fetal losses which is common in PCOS.

CONCLUSION: Pioglitazone have beneficial reproductive and metabolic effect in patients with PCOS. As it restores menstrual cycle regularity, achieve better ovulatory rate and correct hyperandrogenism and insulin resistance.. Also because of its good effect on endothelial cells it may come up as preventive therapy for decreasing cardiovascular risk and type 2 diabetes, but this require long term studies.

\section{REFERENCES:}

1. Asunction M, Caivo RM, San Millanet all. A Prospective study of the polycystic ovary syndrome in unselected

2. Caucasian women.J clin endocrinal Metab 2000:88(7) 2434-8.Journal of clinical endocrinal metab 2004, 89(6)2745-9

3. Brettenhaler N, De Geyter C, Huber PR et al. Effect of insulin sensitizer pioglitazone on IR, hyperandrogenism and ovulatory cydfunction in women with PCOS, J endocrine metab2004

4. Froment P, Touraine $P$; Thiozolidinediones and fertility in PCOS PPAR Res 2006

5. Gonzalez C ; response of serum androgen and insulin resistannce to metformin and pioglitazone in obese PCOS J of endocrine metab 2005

6. Ciampalli M, Lanzone A, Insulin and polycystic ovary; a new look to old subject. Gynecolendocrinol 1998

7. Yilmez M, Karalas A et al J ENDOCRINAL INV 2005, 28(11) 1003-8

8. Ibanzer L, Hum Reprod 2003 18(1)57-58

9. Setto- young D, Palo M J clinendocrinolmetab 2005, 90(11) 6099-105

10. Glueck CJ, Moreira A, Goldenberg et al Hum Reprod 2003, 18

11. ParadisiÂA, SteinbergÂA HO, HempflingÂA, CroninÂA, HookÂ G, ShepardÂ MK, etÂ al.ÂP Polycystic ovary syndrome is associated with endothelial dysfunction. Circulation. 2001;103:1410â€"1415

12. ChristianÂ RC, DumesicÂ DA, BehrenbeckÂ T, ObergÂAL, SheedyÂ PF, FitzpatrickÂ LA. Prevalence and predictors of coronary artery calcification in women with polycystic ovary syndrome. J ClinEndocrinolMetab. 2003;88:2562â€"2568

13. OrioÂ F, PalombaÂ S, CascellaÂ T, De SimoneÂA, Di BiaseÂ S, RussoÂ T, etÂ al.Â Early impairment of endothelial structure and function in young normal-weight women with polycystic ovary syndrome. J ClinEndocrinolMetab. 2004;89:4588â€“4593 
14. .KravaritiÂA, NakaÂ KK, KalantaridouÂ SN, KazakosÂ N, KatsourasÂ CS, Makrigiannakis $\hat{A} A$, etÂ al.Â Predictors of endothelial dysfunction in young women with polycystic ovary syndrome. J ClinEndocrinolMetab. 2005;90:5088â€“5095

15. ShroffÂ R, KerchnerÂ A, MaifeldÂ M, Van BeekÂ EJ, JagasiaÂ D, DokrasÂ A. Young obese women with polycystic ovary syndrome have evidence of early coronary atherosclerosis. J ClinEndocrinolMetab. 2007;92:4609â€"4614

16. .Diamanti-KandarakisÂ E, AlexandrakiÂ K, ProtogerouÂA, PiperiÂ C, PapamichaelÂ C, AessoposÂA, etÂ al.Â Metformin administration improves endothelial function in women with polycystic ovary syndrome. Eur J Endocrinol. 2005;152:749â€“756

17. .OrioÂF, PalombaÂ S, CascellaÂ T, De SimoneÂ B, MangusoÂF, SavastanoÂ S, et $\hat{A}$ al.Â Improvement in endothelial structure and function after metformin treatment in young normal-weight women with polycystic ovary syndrome: results of a 6-month study. JÂ ClinEndocrinolMetab. 2005;90:6072â€"6076 